Check for updates

Cite this: RSC Adv., 2017, 7, 33486

\title{
Research on cellulose nanocrystals produced from cellulose sources with various polymorphs
}

\author{
Jie Gong, (D) ab Jun Li, (D) *ab Jun Xu, (D) ab Zhouyang Xiang ${ }^{\mathrm{ab}}$ and Lihuan Mo ${ }^{\mathrm{ab}}$
}

This study investigated the impact of cellulose polymorphs on cellulose nanocrystals (CNCs) preparation. Cellulose samples with different types of polymorphs (cellulose I, cellulose II, and cellulose III) were prepared. Subsequently, CNCs were obtained from the above cellulose samples by sulfuric acid hydrolysis. The initial cellulose and CNCs were characterized by X-ray diffraction (XRD), thermo gravimetric analysis (TGA), scanning electronic microscopy (SEM) and particle size distribution (PSD). A comparative study showed that the properties of CNCs were closely related to the polymorphs of initial cellulose. The original polymorphs of cellulose I and cellulose II were retained, while that of cellulose III was converted back to cellulose I during the sulfuric acid hydrolysis process of CNCs preparation. For cellulose I and cellulose II, the crystallinity of both corresponding CNCs continuously increased with the increase of sulfuric acid concentration, until reaching a maximum at approximately $84.0 \%$, while the crystallinity of CNCs obtained from cellulose III was slightly affected by sulfuric acid concentration. The yields of CNCs obtained from cellulose II and cellulose III with $61 \mathrm{wt} \% \mathrm{H}_{2} \mathrm{SO}_{4}$ were extremely high, reaching $46.8 \%$ and $43.4 \%$, respectively. Smaller CNCs particles with short-length were obtained by sulfuric acid hydrolysis of cellulose II. In addition, the properties (yield, dimension and thermal degradation behavior) of CNCs obtained from cellulose III were more susceptible to the acid concentration compared with those of CNCs obtained from cellulose I.

Received 3rd June 2017

Accepted 25th June 2017

DOI: $10.1039 / c 7 r a 06222 b$

rsc.li/rsc-advances

\section{Introduction}

As a plentiful renewable natural resource on earth, the efficient utilization of cellulose is becoming increasingly important. Recently, cellulose fibers subjected to acid hydrolysis, yield nanoscale rod-like crystalline residues, which are called cellulose nanocrystals (CNCs) ${ }^{1,2}$ or nanocrystalline cellulose (NCC). ${ }^{3,4}$ CNCs can be extracted from a great variety of sources, including wood pulps, ${ }^{5}$ cotton, ${ }^{6}$ sisal, ${ }^{7}$ hemp,${ }^{8}$ flax,${ }^{8}$ microcrystalline cellulose (MCC), ${ }^{9,10}$ and so on. As a nano-material, CNCs exhibits many appealing properties, such as high crystallinity, large aspect ratio, biodegradability, nano-dimensions, light weight, and large surface area. ${ }^{11-13}$ Owing to these features, it has shown great promise in many fields, including reinforcing fillers for composite materials, ${ }^{14}$ stabilizing agents for emulsions, ${ }^{15}$ templates for the chemical synthesis of inorganic materials, ${ }^{16}$ and so on. The studies on CNCs have gained increasing attention in recent years.

Acid hydrolysis is one of the most commonly adopted techniques to prepare CNCs. Several acids have been used, such as sulfuric acid, ${ }^{6,10,11}$ phosphoric acid $^{17}$ and hydrochloric acid..$^{18}$

${ }^{a}$ State Key Laboratory of Pulp and Paper Engineering, South China University of Technology, Guangzhou 510640, China. E-mail: ppjunli@scut.edu.cn; Tel: +86 13503012206

${ }^{b}$ United Lab of Plant Resources Chemistry and Chemical Engineering, South China University of Technology, Guangzhou 510640, China
Among these acids, sulfuric acid has been commonly used for CNCs extraction because of the good stability of the resulting suspensions. ${ }^{2}$ However, the use of sulfuric acid has a number of drawbacks, such as vulnerable to thermal degradation, ${ }^{2}$ low yield and long reaction time. ${ }^{4}$ To overcome these shortcomings, some eco-friendly approaches, including purely physical method of ultrasonication, ${ }^{5,10}$ enzyme-assisted hydrolysis, ${ }^{6}$ as well as combined processes ${ }^{17}$ have been developed to assist acid hydrolysis in the past decades. However, a number of limitations such as properties prediction, high cost, and durability still need to be considered. ${ }^{4}$

Typical sulfuric acid hydrolysis currently employed for the CNCs preparation under strictly controlled the conditions of temperature, time, acid concentration, acid-to-cellulosic fiber ratio, and agitation. The temperature can range from room temperature up to $70{ }^{\circ} \mathrm{C}$ and the corresponding hydrolysis time can be varied from 30 min to several hours depending on the temperature. ${ }^{2}$ The concentration of sulfuric acid does not vary much from a typical value of $64 \mathrm{wt} \%$ in most studies. ${ }^{1,11,19}$ Thus, the most suitable sulfuric acid concentration has been thought to be $63-65 \mathrm{wt} \%$. The acid-to-cellulose ratio can be varied from $10: 1$ to $20: 1\left(\mathrm{~mL} \mathrm{~g}^{-1}\right){ }^{6,12,20}$

Cellulose has both crystalline and amorphous regions. In crystalline domains, the molecular orientation and hydrogenbonding network can vary widely, which can give rise to cellulose polymorphs. ${ }^{21}$ There are four polymorphs of cellulose, 
namely, cellulose I, cellulose II, cellulose III, and cellulose IV.,13,21 Native cellulose has been thought to have the polymorph of cellulose I (cellulose $\mathrm{I}_{\alpha}$ and cellulose $\mathrm{I}_{\beta}$ ). Cellulose $\mathrm{I}_{\alpha}$ exists in a triclinic $P 1$ unit cell $(a=0.672 \mathrm{~nm}, b=0.596 \mathrm{~nm}, c=$ $\left.1.040 \mathrm{~nm}, \alpha=118.1^{\circ}, \beta=114.8^{\circ}, \gamma=80.4^{\circ}\right),{ }^{22}$ whereas cellulose $\mathrm{I}_{\beta}$ exists in a monoclinic $P 2_{1}$ unit cell $(a=0.778 \mathrm{~nm}, b=$ $\left.0.820 \mathrm{~nm}, c=1.038 \mathrm{~nm}, \gamma=96.5^{\circ}\right) .{ }^{23}$ Both cellulose chains of $\mathrm{I}_{\alpha}$ and $\mathrm{I}_{\beta}$ adopt parallel configurations. ${ }^{2}$ Cellulose II can be obtained by chemical regeneration or mercerization of nature cellulose.,24 The chains of cellulose II are in an antiparallel configuration, and having a monoclinic $P 2_{1}$ unit cell $(a=$ $\left.0.810 \mathrm{~nm}, b=0.903 \mathrm{~nm}, c=1.031 \mathrm{~nm}, \gamma=117.1^{\circ}\right) .{ }^{25}$ In contrast to cellulose I, cellulose II has a more stable structure, which makes it preferable for various applications. ${ }^{26}$ If cellulose I or cellulose II is exposed to ammonia or various amines, cellulose III is formed upon removal of the swelling agent. ${ }^{\text {24,27 }}$ Upon generated from cellulose I or cellulose II, it can be distinguished into two forms, cellulose $\mathrm{III}_{\mathrm{I}}$ and cellulose $\mathrm{III}_{\mathrm{II}}{ }^{24}$ The unit cell of Cellulose $\mathrm{III}_{\mathrm{I}}$ is monoclinic $P 2_{1}(a=0.445 \mathrm{~nm}, b=0.785 \mathrm{~nm}, c=$ $\left.1.031 \mathrm{~nm}, \gamma=105.1^{\circ}\right) .^{28}$ Cellulose III is reactive crystalline cellulose, which is usually used as a precursor of many cellulose derivatives. ${ }^{29}$

In crystalline regions of cellulose, the cellulose chains are tightly packed together and stabilized by strong and very complex hydrogen-bond network. When acid is used as a hydrolyzing agent, the amorphous regions are attacked preferentially by acid and hydrolyzed first, while the crystalline regions present higher resistance to acid attack. ${ }^{2}$ As a result, the amorphous regions of cellulose are removed by acid hydrolysis and the crystalline domains are left to form highly crystalline cellulose. $^{2}$

Owing to the differences in the kinetics of acid hydrolysis between polymorphs of cellulose, the polymorphic transformation of initial cellulose may accelerate the sulfuric acid hydrolysis, and affect the properties of the CNCs. Many studies have referred to the effects of sulfuric acid concentration on CNCs prepared from raw cellulosic materials with the polymorph of cellulose $\mathrm{I}^{\mathbf{1 0 , 1 2 , 3 0}}$ In consideration of the effects of polymorphs, the typical sulfuric acid concentration (64 wt\%) for cellulose I may not be optimal for cellulose II or cellulose III. Thus, in this work, three different polymorphs have been identified for cellulose I, cellulose II, and cellulose III. A comparative study on the properties of CNCs derived from the above cellulose samples by sulfuric acid hydrolysis in various acid concentration $(58,61$, and $64 \mathrm{wt} \%)$ was demonstrated.

\section{Experimental methods}

\section{Materials}

Commercial MCC power (Avicel PH-101, particle size: $\sim 50 \mu \mathrm{m}$ ) purchased from SIGMA-ALDRICH (USA) was used as cellulose materials. All other chemicals, such as sulfuric acid, sodium hydroxide, and ethylenediamine (EDA) were of analytical grade. All cellulose samples were vacuum dried to remove the moisture before use.

\section{Preparation of cellulose samples with various polymorphs}

MCC was used as cellulose I directly. Cellulose II was prepared by immersing MCC in $18.5 \mathrm{wt} \% \mathrm{NaOH}$ solution at room temperature for $1.5 \mathrm{~h}$, and then washing with distilled water thoroughly. ${ }^{31}$ Cellulose III was obtained by soaking MCC in EDA at room temperature for one day, and then washed with methanol for several times to remove EDA completely. ${ }^{27}$ Excess methanol was removed by volatilization under a vacuum atmosphere.

\section{CNCs preparation}

CNCs were obtained by a controlled sulfuric acid hydrolysis, according to the protocol described elsewhere. ${ }^{6,20,21}$ Briefly, a total of $2 \mathrm{~g}$ above treated cellulose samples (cellulose I, cellulose II, and cellulose III, based on dry weight) were hydrolyzed in $30 \mathrm{~mL} \mathrm{H}_{2} \mathrm{SO}_{4}$ solution of given concentrations $(58,61$, and $64 \mathrm{wt} \%)$. The reaction was performed in a water bath under continuous stirring at $45{ }^{\circ} \mathrm{C}$ for $40 \mathrm{~min}$. The hydrolysis was stopped by adding a large amount of distilled water $(600 \mathrm{~mL})$. After the cellulose powder precipitated, the supernatant was decanted. The resulting suspension was centrifuged (10 $000 \mathrm{rpm}$ ) at $25{ }^{\circ} \mathrm{C}$ for $15 \mathrm{~min}$, and the supernatant fluid was discarded to remove $\mathrm{H}_{2} \mathrm{SO}_{4}$. This centrifugation process was repeated several times until the supernatant was not clear. The cloudy suspension was collected and then dialyzed against distilled water using dialysis bags until the $\mathrm{pH}$ of the suspension reached a constant value. A portion of the CNCs suspension was stored in a refrigerator at $4{ }^{\circ} \mathrm{C}$, and the other was freeze-dried. The residues generated in above centrifugation processes were also collected, dialyzed, and freeze-dried. The CNCs samples derived from cellulose I were denoted as CNC-I, or denoted as CNC-I-58, CNC-I-61 and CNC-I64 , respectively, based on various acid concentrations. Where I denotes the polymorph of corresponding initial cellulose, and 58,61 , and 64 denotes the acid concentration (wt\%). Similarly, the CNCs samples derived from cellulose II and cellulose III were denoted as CNC-II (or CNC-II-58, CNC-II-61, and CNC-II64) and CNC-III (or CNC-III-58, CNC-III-61, and CNC-III-64), respectively. The same principle was employed for the denotations of residues generated in centrifugation processes.

\section{X-ray diffraction analysis}

The XRD patterns for all cellulose and CNCs samples were obtained with an X-ray diffractometer (D8 ADVANCE, Bruker, Germany) using a $\mathrm{Cu} \mathrm{K} \alpha$ radiation at $40 \mathrm{kV}$ and $100 \mathrm{~mA}$. Scattered radiation was detected in the range of $2 \theta=5-50^{\circ}$ at a scan rate of $4^{\circ} \mathrm{min}^{-1}$.

XRD data were analyzed using the MDI Jade 5.0 software. The crystallinity and crystallite size were calculated using the previous method. ${ }^{21,26}$ Briefly, the crystallinity was determined based on the ratio of crystalline region's area to the total area in the XRD spectra. The crystallite sizes were estimated using the Scherrer's equation:

$$
\beta=\frac{0.9 \lambda}{\tau \cos \theta}
$$


where $\beta$ is the full width at half maximum (FWHM) of XRD peaks, $\lambda$ is the wavelength of incident X-rays, $\tau$ is the crystallite sizes, $\theta$ is the diffraction angles corresponding to the planes.

\section{Calculations of CNCs yield and residue yield}

CNCs yield was determined by weighting a $20 \mathrm{~mL}$ aliquot of the suspension after oven-drying for overnight. Both CNCs yield and residue yield were expressed as \% of corresponding initial cellulose and calculated using the following equations:

$$
\text { CNCs yield }(\%)=\frac{M_{2} \times V_{1}}{M_{1} \times V_{2}} \times 100 \%
$$

where $M_{1}$ is the total mass of initial cellulose samples, $M_{2}$ is the mass of oven-dried CNCs at $105{ }^{\circ} \mathrm{C}, V_{1}$ is the total volume of CNCs suspension, and $V_{2}$ is the volume of the CNCs suspension for oven-drying, $20 \mathrm{~mL}$.

$$
\text { Residue yield }(\%)=\frac{M_{3}}{M_{1}} \times 100 \%
$$

where $M_{1}$ is the total mass of initial cellulose samples, $M_{3}$ is the mass of the residues.

\section{Scanning electron microscopy observation}

A droplet of the diluted CNCs suspension $(0.01 \% \mathrm{w} / \mathrm{v}$, sonicated $)$ was deposited in a mica plate. Then the excess liquid was removed by filter paper, and subsequently dried in air. All samples were coated with gold before observation. The SEM images of CNCs were observed by using a field-emission scanning electron microscope (MERLIN Compact, Zeiss, Germany).

\section{Particle size distribution determination}

The PSD and average particle size of CNCs were determined by dynamic laser scattering (DLS) using a nano-particle analyzer (SZ-100Z, Horiba, Japan). All CNCs samples were sonicated in an ultrasonic processor at the power of $200 \mathrm{~W}$ for $180 \mathrm{~s}$ before measuring.

\section{Thermo gravimetric analysis}

TGA was performed using a TGA instrument (Q500, America). The measurements were carried out under nitrogen atmosphere. The weight of all samples was varied from 5 to $10 \mathrm{mg}$. The temperature was increased from room temperature to $600{ }^{\circ} \mathrm{C}$ at a heating rate of $20^{\circ} \mathrm{C} \mathrm{min}^{-1}$. Two tangents from the TG curve, one before inflection caused by the degradation, and the other from the cellulose degradation step, intercrossed. The point of intersection could be explained as the onset degradation temperature. ${ }^{13}$

\section{Results and discussion}

\section{Polymorphic transformation analysis}

XRD patterns of initial cellulose were shown in Fig. 1 (cellulose I (a), cellulose II (b), and cellulose III (c)). For XRD pattern of cellulose I, the well-known diffraction peaks at $2 \theta$ around $14.5^{\circ}$, $16.5^{\circ}$ and $22.5^{\circ}$, were attributed to the planes of $\left(\begin{array}{lll}1 & \overline{1} & 0\end{array}\right),\left(\begin{array}{lll}1 & 1 & 0\end{array}\right)$ and $\left(\begin{array}{ll}2 & 0\end{array}\right)$, respectively, all of which were in agreement with the
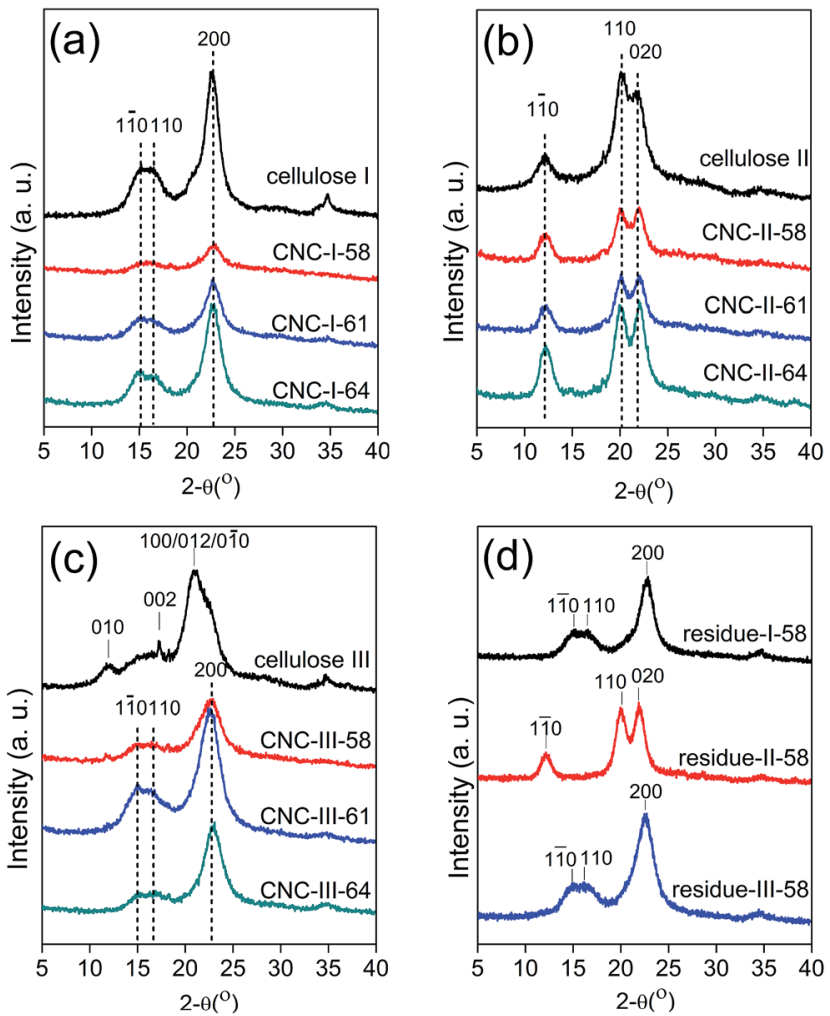

Fig. 1 XRD patterns for cellulose I and CNC-I (a), XRD patterns for cellulose II and CNC-II (b), XRD patterns for cellulose III and CNC-III (c), and XRD patterns for residues generated in the centrifugation process of $\mathrm{CNCs}$ preparation in $58 \mathrm{wt} \% \mathrm{H}_{2} \mathrm{SO}_{4}$ (d).

characteristic diffraction peaks of cellulose I. ${ }^{32}$ For XRD pattern of cellulose II, the diffraction peaks at $2 \theta$ around $12.0^{\circ}, 20.0^{\circ}$ and $22.0^{\circ}$, corresponding to the ( $\left.\begin{array}{lll}1 & \overline{1} & 0\end{array}\right),\left(\begin{array}{lll}1 & 1 & 0\end{array}\right)$ and $\left(\begin{array}{lll}0 & 2 & 0\end{array}\right)$ planes, respectively, were signed to cellulose II. ${ }^{32}$ For XRD pattern of cellulose III, weak peaks ( $2 \theta$ around $\left.14.5^{\circ}, 16.5^{\circ}\right)$ of cellulose I were detected. However, the diffraction peaks at $2 \theta$ around $11.7^{\circ}, 17.3^{\circ}$ and $21.0^{\circ}$, corresponding to the $\left(\begin{array}{lll}0 & 1 & 0\end{array}\right)$ planes, $\left(\begin{array}{lll}0 & 0 & 2\end{array}\right)$ planes and a composite of ( $\left(\begin{array}{lll}1 & 0 & 0\end{array}\right),\left(\begin{array}{lll}0 & 1 & 2\end{array}\right),\left(\begin{array}{lll}1 & \overline{1} & 0\end{array}\right)$ planes, respectively, which attributed to the typical cellulose $\mathrm{III}_{\mathrm{I}}$ were more obvious. ${ }^{32}$ Therefore, the conversions of polymorphs from the original cellulose I to cellulose II and cellulose III were successfully achieved.

XRD patterns of the CNC-I, CNC-II, CNC-III were demonstrated under that of corresponding initial cellulose in Fig. 1, respectively. XRD patterns of CNC-I (Fig. 1a), as well as CNC-III (Fig. 1c), exhibited characteristic diffraction peaks of (1 $1 \overline{1} 0),(11$ $0)$ and $\left(\begin{array}{lll}2 & 0 & 0\end{array}\right)$ planes, which were in agreement with the diffraction peaks of cellulose I. CNC-II (Fig. 1b) obtained from cellulose II exhibited characteristic diffraction peaks of cellulose II. It indicated that only the polymorph of cellulose III had changed during CNCs preparation. A similar result of polymorphic transformation has been observed, in the case of residue-III-58. The probable explanation was that, during cellulose hydrolysis with sulfuric acid, the amorphous regions were attacked by acid and hydrolyzed first, meanwhile the crystalline regions swelled, which might lead to a rearrangement of the cellulose crystalline structure. Several cases of the 
conversion of cellulose III to cellulose I also have been reported in previous studies. ${ }^{33}$ Cellulose III can be converted back to its parent structure by a heat treatment or under a humid environment. ${ }^{2,33}$ For cellulose II, the polymorph of corresponding CNCs had not changed because the conversion of cellulose I to cellulose II has been widely considered to be irreversible.

\section{Crystallinity analysis}

The results of crystallinity were shown in Fig. 2. It can be seen that, the crystallinity of cellulose I, cellulose II, and cellulose III were $84.5 \%, 50.0 \%$, and $69.4 \%$, respectively. It indicated that the polymorphic transformation may lead to a decrease in crystallinity. Both crystallinity of the residue-II- 58 and residueIII-58 were increased compared to their corresponding initial cellulose. That was because most of hemicelluloses and cellulose in amorphous region was removed by sulfuric acid hydrolysis. But for cellulose I, the crystallinity of residue-I-58 has not increased. It was $81.4 \%$, even slightly lower than that of the initial cellulose, $84.5 \%$. It indicated that the crystallinity increased by sulfuric acid hydrolysis reached a maximum value, as pointed out by the dash line in Fig. 2, around $84.0 \%$, and a deeper hydrolysis would not increase the crystallinity. It was explained by the fact that, during the cellulose sulfuric acid hydrolysis, cellulose in amorphous region was hydrolyzed, at the same time, partially of cellulose molecules in crystalline regions reacted with sulfuric acid and became dissolved. If the crystalline region dominates the cellulose, both processes will take place in a balance.

The results of the crystallinity of CNC-I samples showed that, when the concentration of sulfuric acid was $58 \mathrm{wt} \%$, the crystallinity was quite low, only $49.3 \%$. The crystallinity was increased to $68.1 \%$ with the sulfuric acid concentration increasing to $61 \mathrm{wt} \%$. When the sulfuric acid concentration was further increased to $64 \mathrm{wt} \%$, the crystallinity was $81.3 \%$, very close to that of corresponding initial cellulose, $84.5 \%$, which reached the maximum value as described above, around $84.0 \%$. It indicated that the crystallinity of CNC-I was continuously increased with the increase in sulfuric acid concentration. The probably explanation was that, in a low sulfuric acid concentration, the cellulose crystalline regions were poorly swelled,

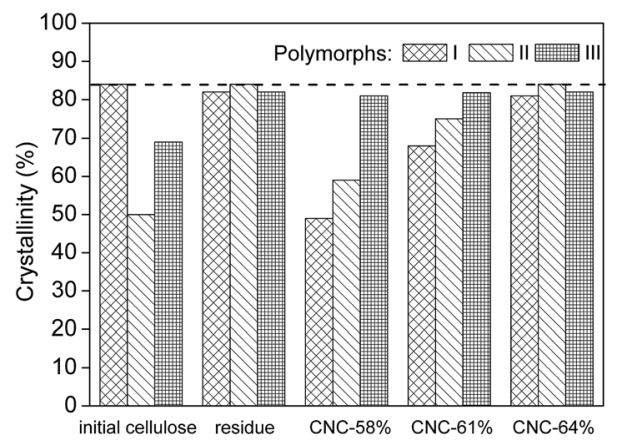

Fig. 2 Crystallinty of CNCs, initial cellulose samples, and the residues generated in the centrifugation process of CNCs preparation in $58 \mathrm{wt} \%$ $\mathrm{H}_{2} \mathrm{SO}_{4}$. and the degree of hydrolysis reaction was low. Cellulose in crystalline regions which has not subjected to sulfuric acid, was remained in the residues of acid hydrolysis, whereas cellulose in amorphous regions, which has preferentially hydrolyzed by sulfuric acid, was introduced the negatively charged sulfate half ester groups onto its outer surface, and formed CNCs particles with a low crystallinity. As the sulfuric acid concentration increased, cellulose crystalline regions were gradually swelled. More cellulose in crystalline regions reacted with sulfuric acid. At the same time, cellulose in amorphous regions has been hydrolyzed, and removed by sulfuric acid hydrolysis completely. As a result, cellulose in crystalline regions which crystallinity was higher, was disintegrated, and turned into high crystallinity CNCs particles. In the cases of CNC-II, it also follows the similar trends as that of CNC-I. It means that we can prepare the CNCs with various crystallinity by controlling the concentration of sulfuric acid. This find has important value in applications, because crystallinity is one of the most important properties of CNCs, which may affect its mechanical, chemical and thermal properties., ${ }^{919}$

Unlike CNC-I and CNC-II, the crystallinity of CNC-III were slightly affected by sulfuric acid concentration, all of which were close to the maximal value (around $84.0 \%$ ). This result may be attributed to the excellent reactivity of cellulose III, which led to a deeper hydrolysis. On the other hand, a rearrangement of molecular chains might also result in a higher crystallinity, during the polymorphic transformation (III to I).

The average crystallite sizes of initial cellulose, CNCs, and residues were calculated and listed in Table 1. For cellulose I, the trend of average crystallite size was similar to that of crystallinity, which was continuously increased with the increase in sulfuric acid concentration. The probable explanation was that, there was an association between average crystallite size and crystallinity in nature cellulose. Cellulose with lower crystallinity might have smaller crystallites in its crystalline regions. In the case of cellulose II, the average crystallite sizes of all CNCs samples were approximately $5.5 \mathrm{~nm}$, very close to the result of Jin et al., ${ }^{21} 5.1 \mathrm{~nm}$. It indicated that sulfuric acid concentration had a limited effect on average crystallite size of CNC-II. In case of CNC-III, it also follows the similar trend as that of CNC-I, but the average crystallite sizes of CNC-III were smaller than those of CNC-I. This find illustrated that, the molecular chains of cellulose III were rearranged, during the sulfuric acid

Table 1 The average crystallite sizes of CNCs, initial cellulose samples, and the residues generated in the centrifugation process of $\mathrm{CNCS}$ preparation in $58 \mathrm{wt} \% \mathrm{H}_{2} \mathrm{SO}_{4}$

\begin{tabular}{lllr}
\hline & \multicolumn{3}{l}{ Average crystallite sizes (nm) } \\
\cline { 2 - 4 } Samples & $\mathrm{I}$ & $\mathrm{II}$ & $\mathrm{III}$ \\
\hline Initial cellulose & 4.4 & 3.4 & 17.9 \\
Residue-58 & 4.4 & 5.9 & 3.8 \\
CNC-58 & 3.5 & 5.5 & 2.9 \\
CNC-61 & 4.1 & 5.6 & 3.3 \\
CNC-64 & 4.2 & 5.5 & 3.6
\end{tabular}


hydrolysis. And such rearrangement might lead to smaller crystallite sizes. The method that evidence polymorphic transformation via analysis of average crystallite sizes was also adopted by Jin et $a .^{21}$

\section{CNCs yield}

The yields of each CNCs and residues were shown in Fig. 3 (CNCs, (a) and residues, (b)). Clearly, sulfuric acid concentration had a marked effect on the yield of CNCs. When the concentration of sulfuric acid was $58 \mathrm{wt} \%$, the initial cellulose was poorly hydrolyzed, which resulted in a low CNCs yield. On the contrary, the yields of residues were high, because a large amount of the initial cellulose in crystalline regions has not reacted with the sulfuric acid. Therefore, a too low sulfuric acid concentration was unfavorable for CNCs preparation. When the acid concentration was increased to $61 \mathrm{wt} \%$, all of CNCs yields were increased. However, the yields of CNC-II and CNC-III had a remarkable improvement, reaching $46.8 \%$ and $43.4 \%$, respectively, much higher than that of CNC-I, which was only $24.9 \%$. On the contrary, the residue yields of cellulose II and cellulose III were significantly decreased. They were reduced from $72.0 \%$ to $4.1 \%$, and from $44.3 \%$ to $2.4 \%$, respectively, while that of cellulose I was reduced only from $66.6 \%$ to $48.3 \%$. It indicated that, in $61 \mathrm{wt} \% \mathrm{H}_{2} \mathrm{SO}_{4}$, cellulose I was hydrolyzed incompletely, while cellulose II and cellulose III were hydrolyzed more fully, but not exceedingly, which further disintegrated the initial cellulose into nano-particles and led to a high CNCs yield. In the case of $64 \mathrm{wt} \% \mathrm{H}_{2} \mathrm{SO}_{4}$, there was a decrease in yields of all CNCs, especially in CNC-III. This might attribute to exceedingly hydrolysis, which could lead to

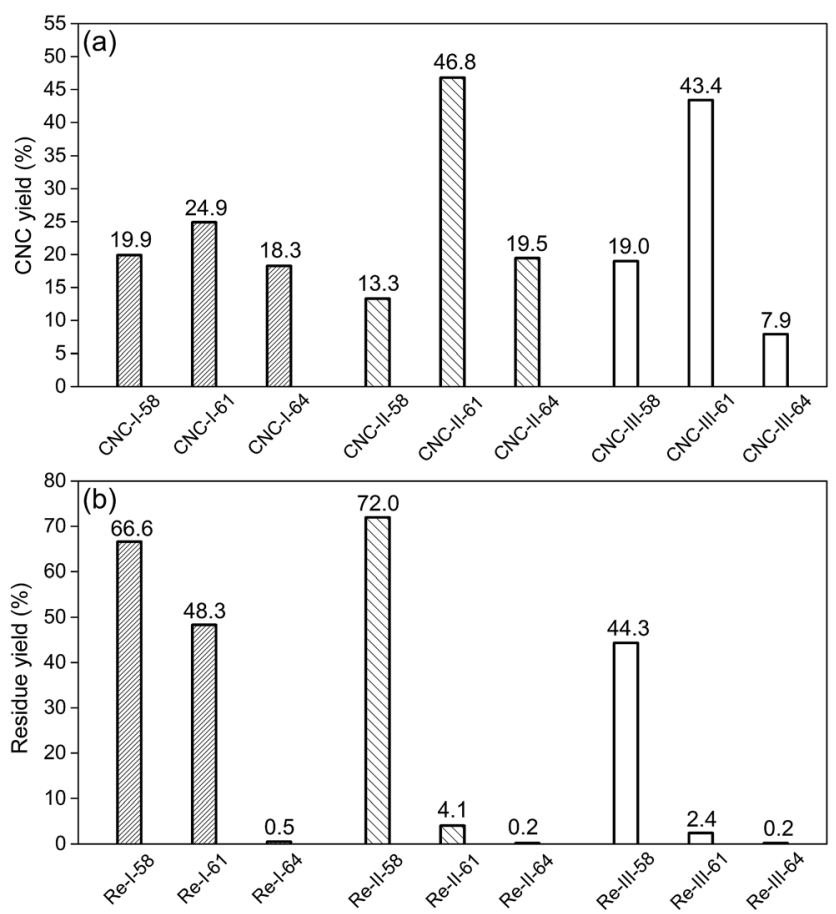

Fig. 3 Yields of CNCs (a), yields of residues obtained in the centrifugation process of CNCs preparation (b). a serious degradation of cellulose to oligosaccharide. Similar trend was reported by Tang et al. ${ }^{\mathbf{1 0}}$ where optimum acid concentrations $(65 \mathrm{wt} \%)$ resulted in higher CNCs yield due to moderate hydrolysis. Beltramino et $a .^{6}{ }^{6}$ have reported that greater CNCs yields were obtained for hydrolysis carried out with $62 \mathrm{wt} \% \mathrm{H}_{2} \mathrm{SO}_{4}$ compared to $64 \mathrm{wt} \%$. Therefore, there were fairly narrow ranges of sulfuric acid concentrations used in the treatment. For cellulose I, the most frequently-used sulfuric acid concentration for CNCs preparation has been thought to be $64 \mathrm{wt} \%$, but for cellulose II and cellulose III, a lower acid concentration ( $61 \mathrm{wt} \%$ ) may be more suitable, because of the good swelling of initial cellulose and relatively high yield of CNCs.

CNCs yield is a key aspect to be analyzed due to its evident impact on the economic cost. Some renewable approaches, such as ultrasonication, enzyme-assisted hydrolysis, have been developed to improve the CNCs yields. Beltramino et al. ${ }^{6}$ studied the effects of pre-treating on cotton linters with cellulase before CNCs preparation. As a result of cellulase treatment, the overall CNCs yield up to $12 \%$ greater. Tang et al. ${ }^{10}$ have reported that CNCs yield reached $40.4 \%$ under a low-intensity ultrasonic-assisted sulfuric acid hydrolysis, while it was only $33.0 \%$ in the absence of ultrasonic treatment. Herein, if establish comparisons between CNCs samples obtained from different initial cellulose, these results discussed above also revealed that the yields of CNC-II and CNC-III were more susceptible to the acid concentration compared to CNC-I. And the yields of CNC-II-61 and CNC-III-61 were much higher than that of CNC-I-61. It indicated that polymorphic transformation of the initial cellulose had a potential for high yields of CNCs, as well as ultrasonic-assisted or enzyme-assisted hydrolysis, if the hydrolysis reaction carry out in a favorable condition.

\section{Morphological and dimensional analysis}

The morphological and dimensional analysis of CNCs were determined by SEM and combined with PSD which would give more insight about the CNCs particles. SEM images were demonstrated in Fig. 4. It can be seen that CNC-I as well as CNCIII particles exhibited a rod-like structure. The length of CNC-III has decreased greatly, from 100-300 nm to $20-100 \mathrm{~nm}$, with the increase in acid concentration. While in the case of CNC-I, negligible differences in morphology and dimension between each CNC-I samples were observed. All of them were 100$300 \mathrm{~nm}$ in length, and $10-30 \mathrm{~nm}$ in width. It indicated that the morphology of CNC-III was more susceptible to the acid concentration compared to CNC-I, although both of them exhibited a rod-like shape. For CNC-II, the morphology was also impervious to the acid concentration. But its length was quite short, only around 10-40 nm. Similar morphology was observed by TEM in previous studies of Jin et al. ${ }^{21}$ and Neto et al. ${ }^{34}$ As explained by Jin et al., ${ }^{21}$ this result may be attributed to the special functions of cellulose mercerization. Briefly, hydroxide ions in the $\mathrm{NaOH}$ solution penetrated into the cellulose crystalline regions and swelled them. Then the cellulose chains rearranged their orientation as hydroxide ions were removed from the lattice of the cellulose matrix. In this process, cellulose 


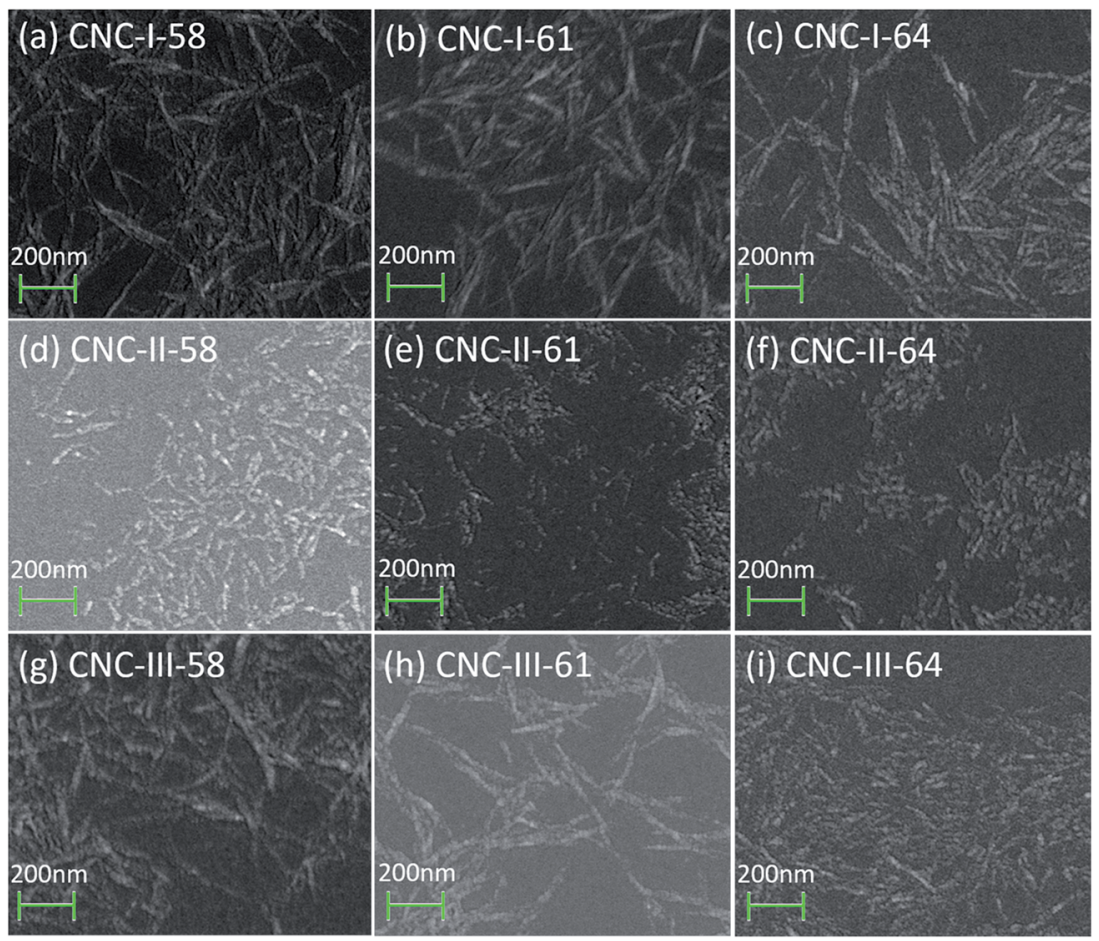

Fig. 4 SEM images of CNCs samples.

chains folded and packed anti-parallel, and formed a more stable structure. As such cellulose structure may lead to the much shorter length of CNCs. Such CNCs with non rod-like structure was also obtained via ultrasonic assisted hydrolysis of MCC with mix acid in previous studies of Wang et al. ${ }^{18}$

The PSD and average particle sizes of CNCs were determined by DLS, which are not good techniques to use on rod-shaped particles because the data calculates particles diameters considering all elements to be spheres. The sizes recorded by DLS can only be taken as a relative value and cannot be compared with that determined by SEM. Still, it can be used to establish comparisons between CNCs samples. ${ }^{21}$ The results of PSD and average particle sizes were demonstrated in Fig. 5 and listed in Table 2, respectively. It can be seen that, polymorphic transformation exerted an important role in the PSD of CNCs.
For all CNCs samples, similar trends were observed, where a marked decrease in particle size range and get more uniform with the increase in acid concentration, although all the curves of PSD exhibited two peaks, which were similar to the previously reported results. ${ }^{10,17}$ But the average particle size of CNC-III was decreased more seriously, from $437 \mathrm{~nm}$ to $123 \mathrm{~nm}$, while that of CNC-I was only decreased from $403 \mathrm{~nm}$ to $218 \mathrm{~nm}$, with the increase in acid concentration. It indicated that, as well as morphology, the dimension of CNC-III were more susceptible to the acid concentration compared to CNC-I. In addition, the polymorphs of the initial cellulose also had great effects on particle size distribution of CNCs. Cellulose II and cellulose III were more likely to obtain CNCs with a narrow particle size distribution. In case of $64 \% \mathrm{H}_{2} \mathrm{SO}_{4}$, the overall particle size range of CNC-I, CNC-II, and CNC-III were $50-840 \mathrm{~nm}, 30-$
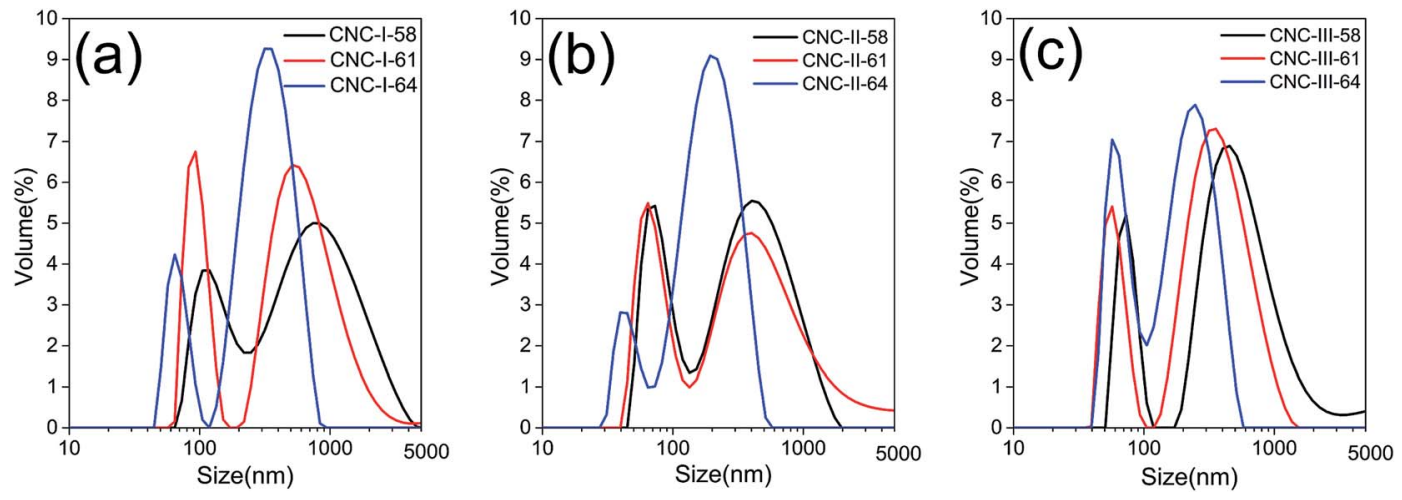

Fig. 5 Particle size distribution of CNC-I (a), CNC-II (b), and CNC-III (c). 
Table 2 The average particle sizes of CNC particles

\begin{tabular}{llll}
\hline & \multicolumn{3}{l}{ Average particle sizes (nm) } \\
\cline { 2 - 4 } Acid concentration $(\%)$ & CNCs-I & CNCs-II & CNCs-III \\
\hline 58 & 403 & 205 & 437 \\
61 & 372 & 197 & 298 \\
64 & 218 & 134 & 123 \\
\hline
\end{tabular}

$580 \mathrm{~nm}$, and $40-580 \mathrm{~nm}$ respectively. And the average particle size of CNC-II and CNC-III were $134 \mathrm{~nm}$ and $123 \mathrm{~nm}$, respectively, smaller than that of CNC-I, $218 \mathrm{~nm}$. It worth mentioning that a remarkable decreased in the particle size range of CNCs would facilitate the reinforcement effect of CNCs in related composites. ${ }^{17}$ These results of PSD were in accordance with the results of SEM analyses (Fig. 4).

The results of SEM and PSD also revealed that polymorphic transformation of initial cellulose before sulfuric acid hydrolysis has important effects on the morphology and dimension of CNCs. If cellulose is converted to cellulose II before sulfuric acid hydrolysis, CNCs particles with shot-length can be easily obtained. If it is converted to cellulose III, smaller CNCs particles which need a harsher hydrolysis in conventional preparation can be obtained in a fairly mild hydrolysis condition, without causing a serious decrease in yield. All these purposes was difficult to achieved in absence of the polymorphic transformation.

\section{Thermal degradation behavior of CNCs}

The TG and DTG curves of cellulose and CNCs were shown in Fig. 6. In a low temperature $\left(<105{ }^{\circ} \mathrm{C}\right)$ range, an initial small weight loss of all samples corresponded to the evaporation of absorbed water. As can be seen in Fig. 6a, the weight losses of initial cellulose samples were extremely similar. The pronounced degradations of all cellulose samples were started at approximately $250{ }^{\circ} \mathrm{C}$, and the temperatures of the maximum degradations of cellulose I, cellulose II and cellulose III were $338{ }^{\circ} \mathrm{C}, 350{ }^{\circ} \mathrm{C}$ and $355^{\circ} \mathrm{C}$, respectively. The degradations were completed at approximately $380{ }^{\circ} \mathrm{C}$. It indicated that although the polymorph has changed, all cellulose samples still had a similar thermal degradation behavior.

The thermal degradation behavior of CNCs was more complicated and quite different from that of cellulose, as shown in Fig. 6b-d. In general, CNCs exhibited a lower degradation temperature, which may be attributed to the introduction of the negatively charged sulfate half ester groups and a larger number of free ends of chains in CNCs caused by the sulfuric acid hydrolysis. ${ }^{\mathbf{1 8 , 3 5}}$ For CNC-I and CNC-II, the thermal degradation behaviors were similar. Two major weight losses, at approximately $105-300{ }^{\circ} \mathrm{C}$, and approximately $300-400{ }^{\circ} \mathrm{C}$ were observed in the DTG curves. It indicated that sulfuric acid
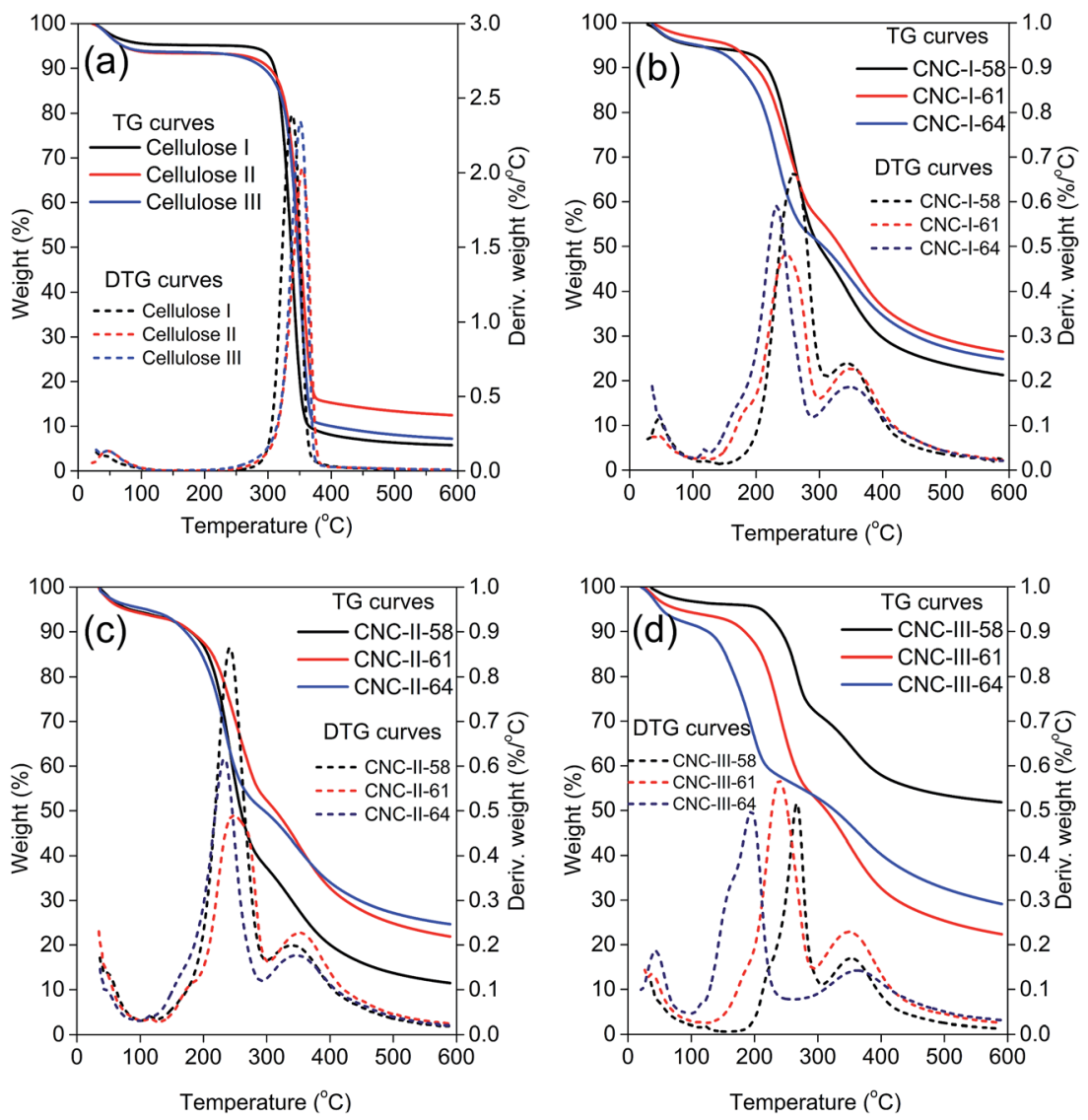

Fig. 6 TG-curves and DTG-curves of initial cellulose (a),CNC-I (b), CNC-II (c), CNC-III (d). 
concentration had a slight effect on thermal degradation behaviors of CNC-I and CNC-II. However, the thermal stabilities of CNC-III-58, CNC-III-61, and CNC-III-64 were quite different from each other in the lower thermal degradation range. Their maximum degradations were at $198{ }^{\circ} \mathrm{C}, 238{ }^{\circ} \mathrm{C}$ and $267{ }^{\circ} \mathrm{C}$, respectively. It indicated that thermal stability of CNC-III was remarkably decreased with the increase in sulfuric acid concentration. The possible explanation was that cellulose III could be hydrolyzed more easily by sulfuric acid, and the degree of hydrolysis was more easily affected by acid concentration.

\section{Conclusion}

CNCs were successfully prepared from the cellulose with different polymorphs (cellulose I, cellulose II, and cellulose III), and their properties were investigated. The results showed that the polymorphic transformation of initial cellulose has a significant effect on CNCs properties. If the polymorph is converted to cellulose II, smaller CNCs particles with shortlength can be easily obtained. The polymorph structure of cellulose III has changed during the sulfuric acid hydrolysis, but if the initial cellulose is converted to cellulose III, the CNCs properties, including yield, dimension and thermal degradation behavior, become more susceptible to the acid concentration. That may facilitate the control of the properties of CNCs, during the hydrolysis process. Moreover, polymorphic transformation of the initial cellulose may also has a potential for high yield, if the sulfuric acid hydrolysis reaction takes place in a favorable condition.

\section{Acknowledgements}

The authors acknowledge the support by the National Water Pollution Control and Treatment Science and Technology Major Project (No. 2014ZX07213001), the Special Support Plan for High-Level Talent Cultivation of Guangdong Province (No. 2014TQ01N603) and Key Laboratory of Pulp and Paper Science \& Technology of Ministry of Education of China project (KF201508).

\section{References}

1 Y. Habibi, Chem. Soc. Rev., 2014, 43, 1519-1542.

2 Y. Habibi, L. A. Lucia and O. J. Rojas, Chem. Rev., 2010, 110, 3479-3500.

3 D. Klemm, F. Kramer, S. Moritz, T. Lindström, M. Ankerfors, D. Gray and A. Dorris, Angew. Chem., Int. Ed., 2011, 50, 54385466.

4 L. Brinchi, F. Cotana, E. Fortunati and J. M. Kenny, Carbohydr. Polym., 2013, 94, 154-169.

5 W. Li, R. Wang and S. Liu, BioResources, 2011, 6, 4271-4281.

6 F. Beltramino, M. B. Roncero, T. Vidal, A. L. Torres and C. Valls, Bioresour. Technol., 2015, 192, 574-581.

7 J. I. Morán, V. A. Alvarez, V. P. Cyras and A. Vázquez, Cellulose, 2008, 15, 149-159.

8 Y. Xu, J. Salmi, E. Kloser, F. Perrin, S. Grosse, J. Denault and P. C. K. Lau, Ind. Crops Prod., 2013, 51, 381-384.
9 S. Cui, S. Zhang, S. Ge, L. Xiong and Q. sun, Ind. Crops Prod., 2016, 83, 346-352.

10 Y. Tang, S. Yang, N. Zhang and J. Zhang, Cellulose, 2014, 21, 335-346.

11 W. Bai, J. Holbery and K. Li, Cellulose, 2009, 16, 455-465.

12 T. Kos, A. Anžlovar, M. Kunaver, M. Huskić and E. žagar, Cellulose, 2014, 21, 2579-2585.

13 Y. Yue, C. Zhou, A. D. French, G. Xia, G. Han, Q. Wang and Q. Wu, Cellulose, 2012, 19, 1173-1187.

14 W. Li, J. Yue and S. Liu, Ultrason. Sonochem., 2012, 19, 479485.

15 F. Cherhal, F. Cousin and I. Capron, Biomacromolecules, 2016, 17, 496-502.

16 Y. Shin and G. J. Exarhos, Mater. Lett., 2007, 61, 2594-2597. 17 Y. Tang, X. Shen, J. Zhang, D. Guo, F. Kong and N. Zhang, Carbohydr. Polym., 2015, 125, 360-366.

18 N. Wang, E. Ding and R. Cheng, Polymer, 2007, 48, 34863493.

19 L. V. Hai, H. N. Son and Y. B. Seo, Cellulose, 2015, 22, 17891798.

20 E. D. M. Teixeira, A. C. Corrêa, A. Manzoli, F. D. L. Leite, C. R. D. Oliveira and L. H. C. Mattoso, Cellulose, 2010, 17, 595-606.

21 E. Jin, J. Guo, F. Yang, Y. Zhu, J. song, Y. Jin and O. J. Rojas, Carbohydr. Polym., 2016, 143, 327-335.

22 Y. Nishiyama, J. Sugiyama, H. Chanzy and P. Langan, J. Am. Chem. Soc., 2003, 125, 14300-14306.

23 Y. Nishiyama, P. Langan and H. Chanzy, J. Am. Chem. Soc., 2002, 124, 9074-9082.

24 R. N. Goldberg, J. Schliesser, A. Mittal, S. R. Decker, A. F. L. O. M. Santos, V. L. S. Freitas, A. Urbas, B. E. Lang, C. Heiss, M. D. M. C. R. D. Silva, B. F. Woodfield, R. Katahira, W. Wang and D. K. Johnson, J. Chem. Thermodyn., 2015, 81, 184-226.

25 P. Langan, Y. Nishiyama and H. Chanzy, Biomacromolecules, 2001, 2, 410-416.

26 P. Mansikkamäki, M. Lahtinen and K. Rissanen, Carbohydr. Polym., 2007, 68, 35-43.

27 X. Chen, J. Chen, T. You, K. Wang and F. Xu, Carbohydr. Polym., 2015, 125, 85-91.

28 M. Wada, H. Chanzy, Y. Nishiyama and P. Langan, Biomacromolecules, 2004, 37, 8548-8555.

29 Y. Habibi and M. R. Vignon, Cellulose, 2008, 15, 177-185.

30 F. Beltramino, M. B. Roncero, A. L. Torres, T. Vidal and C. Valls, Cellulose, 2016, 23, 1777-1789.

31 M. Wada, M. Ike and K. Tokuyasu, Polym. Degrad. Stab., 2010, 95, 543-548.

32 A. D. French, Cellulose, 2014, 21, 885-896.

33 M. Wada, Macromolecules, 2001, 34, 3271-3275.

34 W. P. F. Neto, J. Putaux, M. Mariano, Y. Ogawa, H. Otaguro, D. Pasquini and A. Dufresne, $R S C A d v ., 2016$, 6, 7601776027.

35 D. Y. Kim, Y. Nishiyama, M. Wada and S. Kuga, Cellulose, 2001, 8, 29-33. 